\title{
The isotope effect in the Hubbard model with local phonons
}

\author{
Alexandru Macridin and M. Jarrell \\ University of Cincinnati, Cincinnati, Ohio, 45221, USA
}

(Dated: November 11, 2018)

\begin{abstract}
The isotope effect (IE) in the two-dimensional Hubbard model with Holstein phonons is studied using the dynamical cluster approximation with quantum Monte Carlo. At small electron-phonon (EP) coupling the IE is negligible. For larger EP coupling there is a large and positive IE on the superconducting temperature that decreases with increasing doping. A significant IE also appears in the low-energy density of states, kinetic energy and charge excitation spectrum. A negligible IE is found in the pseudogap and antiferromagnetic (AF) properties at small doping whereas the AF susceptibility at intermediate doping increases with decreasing phonon frequency $\omega_{0}$. This IE stems from increased polaronic effects with decreasing $\omega_{0}$. A larger IE at smaller doping occurs due to stronger polaronic effects determined by the interplay of the EP interaction with stronger AF correlations. The IE of the Hubbard-Holstein model exhibits many similarities with the IE measured in cuprate superconductors.
\end{abstract}

Introduction. Isotope effect (IE) measurements [1] played a significant role in establishing a phononmediated mechanism in conventional superconductors. One of the greatest successes of the Bardeen-CooperSchrieffer (BCS) theory of superconductivity [2] was the ability to explain the IE experimental data. However, the IE in the high $\mathrm{T}_{c}$ materials $[3,4,5]$ is unusual. At optimal doping the IE is small, leading many researchers to argue that phonons do not play an important role in the mechanism of high $\mathrm{T}_{c}$ superconductivity and consequently that its origin must be purely electronic. Nevertheless, the very large IE at small doping clearly shows that phonons play an important role in the physics of the cuprates.

Some investigators have proposed the unusually large IE at small doping may be caused by the vicinity to a quantum critical point, defining a crossover from threedimensional to two-dimensional (2D) physics as the doping decreases [6]. Other studies claim that the IE in cuprates can be attributed to polaron formation [7]. However, most theoretical investigations of the IE in models for high $\mathrm{T}_{c}$ superconductors $[7,8,8]$ ] start with approximations that assume a BCS-type mechanism for $d$-wave pairing and then study the effect of phonon characteristic energy on the superconducting properties. In contrast, the present study employs the dynamical cluster approximation (DCA) 10, 11] and treats, on equal footing, both the EP and electron-electron interactions in a 2D system in the form of the Hubbard-Holstein (HH) model. In previous work employing the DCA 12], the single-band Hubbard model, a generally accepted paradigm for the low energy physics of cuprates [13, 14], shows $d$-wave superconductivity driven by strong electronic correlations. Furthermore, when combined with EP coupling, the superconducting temperature is significantly influenced by polaronic effects which are enhanced in the presence of antiferromagnetic (AF) correlations [15, 16] inherent in the underlying Hubbard model. Here, by polaronic effects we understand renormalization of the single-particle propagator, i.e. reduction of quasiparticle (QP) weight and charge carrier mobility due to EP coupling, and not necessarily the formation of small polarons or a narrow polaronic band. Thus far no investigation of the IE in systems with both strong electronic correlations and EP interaction has been conducted with cluster mean-field methods.

For conventional superconductors the BCS theory predicts that the isotope coefficient $\alpha=-\frac{d \ln T_{c}}{2 d \ln \omega_{0}}=0.5$, where $\mathrm{T}_{c}$ is the superconducting temperature and $\omega_{0}$ the phonon frequency. The HH model with low frequency phonons, displays a positive and unusual IE. For small EP coupling the IE is small $(\alpha \ll 0.5)$ while for values of EP coupling large enough to drive the system in to a regime with strong polaronic effects $\alpha$ can be very large $(\alpha \gg 0.5)$ and $\alpha$ decreases with increasing doping. The IE in the HH model is directly related with the enhancement of polaronic effects with decreasing $\omega_{0}$, as seen in the dependence of the single-particle quantities and charge susceptibility on changes in $\omega_{0}$. A larger IE at smaller doping is due to increased polaronic effects in the presence of stronger $\mathrm{AF}$ correlations as described in a previous investigation [15]. The pseudogap and AF properties display a negligible IE at small doping, whereas the AF susceptibility at intermediate doping increases with decreasing $\omega_{0}$. The size, sign and doping dependence of the IE in the $\mathrm{HH}$ model is similar to experimental data in the cuprates [3, 17, 18].

Formalism. The HH Hamiltonian reads

$$
\begin{aligned}
H & =-t \sum_{\langle i j\rangle \sigma}\left(c_{i \sigma}^{\dagger} c_{j \sigma}+c_{j \sigma}^{\dagger} c_{i \sigma}\right)+U \sum_{i} n_{i \uparrow} n_{i \downarrow} \\
& +\sum_{i} \frac{p_{i}^{2}}{2 M}+\frac{1}{2} M \omega_{0}^{2} x_{i}^{2}+g n_{i} x_{i} .
\end{aligned}
$$

Here $t$ is the nearest-neighbor hopping, $U$ is the on-site Coulomb repulsion between electrons, $\omega_{0}$ is the energy of the dispersionless optical phonons and $g$ is the on-site EP coupling. The dimensionless EP coupling is defined as 
$\lambda=2 g^{2} /\left(2 M \omega_{0}^{2} W\right)$ and represents the ratio of the singleelectron lattice deformation energy $E_{p}=g^{2} /\left(2 M \omega_{0}^{2}\right)$ to half of the electronic bandwidth $W / 2=4 t$. The IE results from the isotopic change of the ions atomic mass $M$ and, since $\omega_{0} \propto M^{-\frac{1}{2}}, \lambda$ is kept fixed and $\omega_{0}$ varies. Results are presented for small phonon frequency $\omega_{0} \leq 0.3 t$ and for $U=8 t$, generally accepted or canonical parameter regimes for studies of this type and in a range that may be appropriate for the cuprates [19].

The DCA 10, 11], a cluster mean-field theory that maps the original lattice model onto a periodic cluster of size $N_{c}=L_{c}^{2}$ embedded in a self-consistent host, is used to study the HH Hamiltonian of (1). Correlations up to a range $L_{c}$ are treated explicitly, while those at longer length scales are described at the mean-field level. With increasing cluster size, the DCA systematically interpolates between the single-site mean-field result and the exact result, while remaining in the thermodynamic limit. Cluster mean-field calculations on the simple Hubbard model [11] or Hubbard model with local phonons [15, 16] have been successful at capturing many key features associated with cuprate materials including a Mott gap and strong AF correlations, $d$-wave superconductivity and the pseudogap phenomenon.

A quantum Monte Carlo (QMC) algorithm [20] modified to perform the sum over both the discrete field used to decouple the Hubbard repulsion and the phonon field $x$ serves as the cluster solver in the DCA. The present calculations are restricted to clusters of size $N_{c}=4$. Low temperature results for larger clusters are inaccessible due to the sign problem in the QMC calculation. The Maximum Entropy method [21] is employed to calculate the real frequency spectra.

The superconducting properties are analyzed using the pairing matrix $M=\Gamma \chi_{0}$. According to the BetheSalpeter equation

$$
\chi=\chi_{0}+\chi_{0} \Gamma \chi=\chi_{0}(1-M)^{-1},
$$

the instability in the two-particle pairing propagator, $\chi$, indicating a possible transition to a superconducting state, occurs when the leading eigenvalue of $M$ reaches one. The corresponding eigenvector $\Phi$ determines the pairing symmetry. $M$ is the product of the pairing interaction vertex $\Gamma$ and the bare bubble $\chi_{0}=G G . \quad \Gamma$ can be seen as an effective paring interaction; $\chi_{0}$ is a convolution of the dressed singleparticle propagators. The effect of EP coupling and $\omega_{0}$ on these two quantities can be studied by looking at $V_{d}=\sum_{K, i \omega ; K^{\prime}, i \omega^{\prime}} \Phi_{d}(K, i \omega) \Gamma\left(K, i \omega ; K^{\prime}, i \omega^{\prime}\right) \Phi_{d}\left(K^{\prime}, i \omega^{\prime}\right)$ and $P_{d}=\sum_{K, i \omega} \Phi_{d}^{2}(K, i \omega) \chi_{0}(K, i \omega)$. They are the respective projections of the interaction vertex $\Gamma$ and $\chi_{0}$ on the subspace spanned by the $d$-wave eigenvector $\Phi$, as discussed in Ref [23].

Results. Previous DCA calculations [15] show that at small doping large changes occur in the system properties

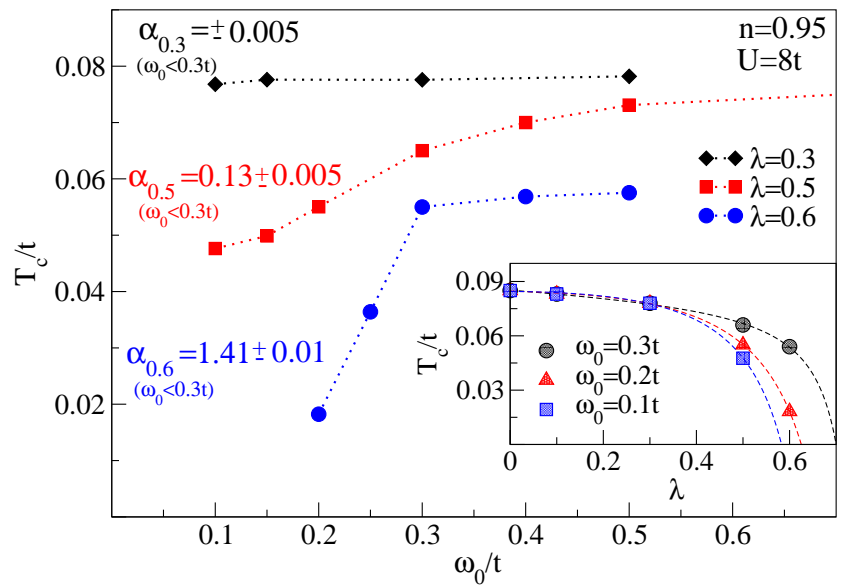

FIG. 1: (color online) $T_{c}$ versus $\omega_{0}$ at $5 \%$ doping for $\lambda=$ $0.3,0.5,0.6$. Inset: $T_{c}$ versus $\lambda$ for $\omega_{0}=0.3 t, 0.2 t, 0.1 t$. The transition to the polaronic regime $(0.5 \lesssim \lambda \lesssim 0.7)$ is accompanied by an abrupt increase of the IE coefficient $\alpha$.

once $\lambda>\lambda_{c} \approx 0.5$. The crossover $(\mathrm{CRO})$ region defining the transition from large to small polaron regime, $0.5 \lesssim$ $\lambda \lesssim 0.7[22$ is characterized by strong polaronic effects, a suppressed but finite $T_{c}$ and a large IE effect. Outside the CRO region, for small EP coupling a negligible IE effect is found, whereas for $\lambda>0.7, T_{c}$ vanishes due to formation of small and heavy polarons. The IE for different values of $\lambda$ is shown in Fig. 1. When $\lambda=0.3$, $T_{c}$ is nearly independent of $\omega_{0}$, and just slightly smaller than $T_{c}$ of the Hubbard model without phonons (see the inset). At $\lambda=0.5$ we find a significant reduction of $T_{c}$ with decreasing phonon frequency $\omega_{0}$ although the IE coefficient $\alpha \approx 0.13$ is much smaller than the BCS value of 0.5. However, for $\lambda=0.6$, i.e. inside the $\mathrm{CRO}$ regime, the IE is very large once $\omega_{0}<0.3 t$. Here the IE coefficient is $\alpha \approx 1.41$.

We find that the IE is decreasing with increasing doping. Fig. 2 (a) shows the logarithm of $T_{c}$ versus the logarithm of $\omega_{0}$ at different dopings when $\lambda=0.5$. To calculate the IE coefficient we use a linear fitting of $\ln \left(T_{c}\right)$ versus $2 \ln \left(\omega_{0}\right)$ for $\omega_{0} \leq 0.4 t$, the slope giving the estimate of $\alpha$ for this study. The IE coefficient dependence on doping is shown in Fig. 2 (b). The decrease of $\alpha$ with increasing doping is in agreement with the notion of significant interplay between the AF correlations, EP interaction and superconductivity [15]. Given stronger $\mathrm{AF}$ correlations at smaller doping, the polaronic effects become more pronounced and hence the IE is larger. It would be interesting to study the doping dependence of the IE for larger values of $\lambda$ (e.g. $\lambda=0.6$ ). Unfortunately at large $\lambda$ and intermediate doping (i.e. $\approx 15 \%$ ) the charge susceptibility of the system becomes extremely large implying strong charge fluctuations that make it very difficult to stabilize the calculation at low temperature. 


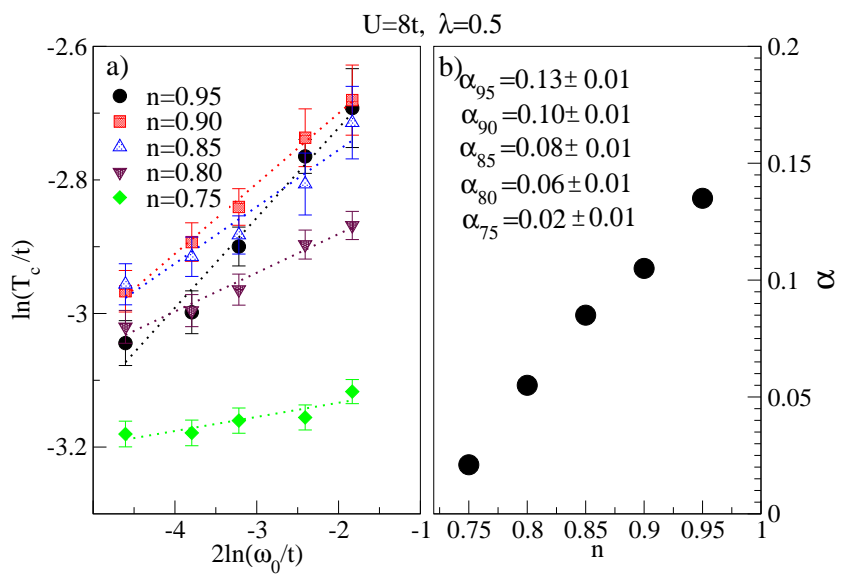

FIG. 2: (color online) (a) $T_{c}$ versus $\omega_{0}$ at $5 \%, 10 \%, 15 \%, 20 \%$ and $25 \%$ doping for $\lambda=0.5$, on a logarithmic scale. A linear fitting of $\ln \left(T_{c}\right)$ versus $2 \ln \left(\omega_{0}\right)$ provides an estimation of IE coefficient $\alpha$ from the slope. (b) $\alpha$ versus the filling $n$ at $\lambda=0.5$. The error bars are of the order of the symbol size. $\alpha$ decreases with increasing doping.

In regard to superconductivity, the EP coupling gives rise to two competing effects [16], an increase of the effective pairing interaction and a renormalization of the single-particle propagator. The first favors superconductivity while the latter opposes it. Keeping the EP coupling fixed and decreasing $\omega_{0}$ results in a similar competition; i.e., an increase of the effective pairing interaction and a stronger renormalization of the single-particle propagator leading to the same overall effect, a reduction of $\mathrm{T}_{c}$. As discussed in Ref. [23], the $d$-wave projected quantities $V_{d}$ and $P_{d}$ provide, respectively, a measure of the effective $d$-wave pairing interaction and renormalization of the single-particle propagator relevant for $d$-wave superconductivity. At low temperature as $\omega_{0}$ decreases $V_{d}$ increases while $P_{d}$ decreases, as shown in Fig. 3 .

In the CRO regime the IE on the superconducting $\mathrm{T}_{c}$ is accompanied by a significant IE in the low energy DOS and in the kinetic energy of the system. For $\lambda=0.6$ when $\omega_{0}$ decreases, the DOS in an energy window of order $\approx \omega_{0}$ around the chemical potential (chosen zero in Fig团 (a)) is suppressed and the kinetic energy at low temperature increases with decreasing $\omega_{0}$, as shown in Fig. (4 (b). Like the projected bubble $P_{d}$ discussed in the previous paragraph, both the DOS and the kinetic energy describe single-particle propreties of the system and are strongly linked to effects on charge carrier mobility and effective mass. Their dependence on $\omega_{0}$ shows that the polaronic effects become stronger as the phonon frequency is reduced, a fact understood by noting that decreased $\omega_{0}$ moves the system closer to the adiabatic regime where the CRO region shrinks to $\lambda_{c}$ and charge carriers should eventually self-trap when $\lambda>\lambda_{c}$ [24].

The magnetic properties are also influenced by the
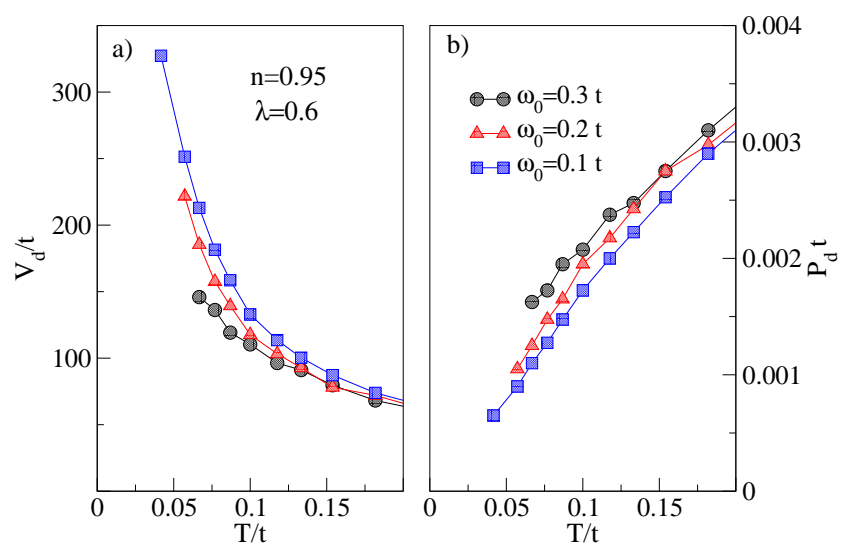

FIG. 3: (color online) (a) The $d$-wave pairing interaction $V_{d}$ and (b) the $d$-wave projected bubble $P_{d}$ versus temperature for different $\omega_{0}$, at $\lambda=0.6$ and $5 \%$ doping. While the pairing interaction increases with decreasing $\omega_{0}$, the decrease of $P_{d}$ results in an overall suppression of $T_{c}$ with decreasing $\omega_{0}$.

phonon energy. At small doping and at half filling the magnetic susceptibilities show very small $\omega_{0}$ dependence, on the order of the error bar, even in the CRO regime (not shown). However, the IE on the Neél temperature $T_{N}$ can be significant near the doping range where $T_{N} \rightarrow 0$. For example, as can be seen in Fig. 4 (c) at 15\% doping, the AF susceptibility increases as $\omega_{0}$ decreases. The pseudogap temperature $T^{*}$ measured as the characteristic temperature for suppression of low energy spin excitations is virtually independent of $\omega_{0}$. It is interesting that when $T^{*}$ is measured as the temperature at which the pseudogap first begins to appear in the DOS, the same conclusion can be drawn. Moreover, one can see in Fig. 4. (a) that the magnitude of the pseudogap measured by the peak-to-peak distance increases weakly as $\omega_{0}$ decreases.

The charge excitation spectrum shows a strong dependence on $\omega_{0}$. In the CRO regime the local (i.e. $r=0$ ) charge excitation spectrum displays a narrow peak in agreement with the assumption that the system tends toward formation of a polaronic band [15]. Moreover, and also in agreement with that assumption, the width of the peak is proportional to $\omega_{0}$ as shown in Fig. 团(d). The low-energy weight of the charge spectrum increases as $\omega_{0}$ decreases and explains the stronger fluctuations in the electronic density and the difficulty in fixing the doping level as $\omega_{0}$ decreases at low temperatures.

Discussions. While the HH Hamiltonian shows an IE in many respects similar to the one seen in cuprates, as we will discuss in the next paragraph, a direct comparison with experiments requires more realistic models which consider the symmetry of the relevant phonon modes. Since the relevant phonons are the ones associated with oxygen vibrations, the explicit inclusion of the oxygen orbitals in the model might be important as well.

However, we find many similarities between the IE in 


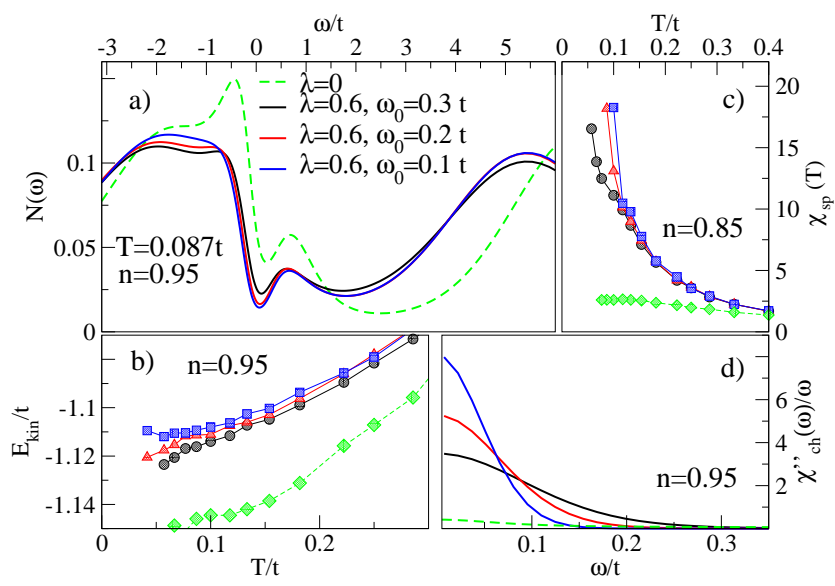

FIG. 4: (color) The black (red, blue) lines represent $\omega_{0}=$ $0.3 t\left(\omega_{0}=0.2 t, \omega_{0}=0.1 t\right)$ results for $\lambda=0.6$ while the green ones show data for $\lambda=0$. (a) DOS $N(\omega)$ and (b) kinetic energy $E_{k i n}=\sum_{k, \sigma} \epsilon(k) n_{k, \sigma}$ at $5 \%$ doping. The low energy DOS and the kinetic energy gain are suppressed as $\omega_{0}$ decreases. c) The AF susceptibility $\chi_{s p}$ at $15 \%$ doping. The AF correlations are enhanced when $\omega_{0}$ decreases. d) The local dynamic charge susceptibility $\chi_{c h}^{\prime \prime}(r=0, \omega) / \omega$ at $5 \%$ doping. The width of low energy peak in the charge excitation spectra is proportional to $\omega_{0}$.

cuprates and our results in the CRO regime. The experiments in cuprates report a decrease of $\alpha$ with increasing doping [3], a negligible IE in the pseudogap and AF properties at small doping [17, 18] and a large IE in the spin glass freezing temperature [25] indicative of an increase of AF correlations at finite doping with decreasing phonon frequency. The large IE seen in the penetration depth shows strong dependence of the Cooper pairs effective mass on phonon frequency [5, 9, 26]. Although we do not address the superconducting state, this may be understood with our findings of strong $\omega_{0}$ dependence of the charge carriers effective mass. The competition between the enhancement of the pairing interaction and the renormalization of the single-particle propagator (discussed in Fig. 3) may be relevant for explaining the negligible IE in the superconducting $\mathrm{T}_{c}$ accompanied by a significant IE in the charge carrier effective mass seen in the optimally doped cuprates [9], since the former is a result of the competition and the latter is a consequence of only the single-particle renormalization. The experiments find an extremely large IE around $8 \%$ doping reportedly linked with stripe formation $[3,27]$. Stripes cannot be addressed with the small $2 \times 2$ clusters since the the stripe phase order parameter is incommensurate with the cluster. However, the strong enhancement of charge fluctuations due to EP coupling and the dependence of charge susceptibility on $\omega_{0}$ suggest a large IE on stripe formation if larger clusters were considered.

Due to the nature of cluster-mean field approximation which neglects spatial correlations outside the cluster range, in the underdoped region where the phase fluctuations (PF) are strong, the polaronic effects' influence on the PF is underestimated. This leads to the expectation of an even larger IE at small doping and a stronger doping dependence of $\alpha$ in calculations which manage to capture longer range correlations. This can be understood by noting that the PF coherence temperature is given by the $\frac{n_{s}}{m^{*}}$, where $n_{s}$ is the superconducting fluid density and $m^{*}$ is the effective mass of the pairs [28]. Thus increased effective mass due to the decrease in $\omega_{0}$ amplifies the $\mathrm{PF}$ and reduces $T_{c}$.

Conclusions. This letter presents results of calculations of the two-dimensional Hubbard model with Holstein phonons using DCA and QMC specifically addressing the IE in light of recent experiments on high $\mathrm{T}_{c}$ materials, including the cuprates. At small EP coupling there is a negligible IE, but for larger EP values, in the CRO regime, a large and positive IE effect is found on the superconducting $\mathrm{T}_{c}$ that becomes stronger at small doping. A significant IE is seen also in single-particle quantities, like the low energy DOS and the kinetic energy, as well as the charge excitation spectrum. This indicates that the IE on $T_{c}$ is directly related with the enhancement of effects such as suppression of QP weight and charge carrier mobility with decreasing $\omega_{0}$. A larger IE at smaller doping is a consequence of an increase in these same effects in the presence of strong AF correlations. A negligible IE is seen in the pseudogap and AF properties at small doping whereas the AF susceptibility at intermediate doping increases with decreasing phonon frequency $\omega_{0}$.

The authors would like to thank Brian Moritz for helpful discussions. This research was supported by NSF DMR-0706379 and CMSN DOE DE-FG02-04ER46129. Supercomputer support was provided by Texas Advanced Computing Center.

[1] E. Maxwell, Phys. Rev. 78, 477 (1950); C. A. Reynolds et al., Phys. Rev. 78, 487 (1950).

[2] J. Bardeen, et al., Phys. Rev. 108, 1175 (1957).

[3] G. Zhao, et al., J. Phys. Cond. Matter 10, 9055 (1998).

[4] G. Zhao, et al., J. Phys.Cond. Matter 13, R569 (2001).

[5] R. Khasanov, et al., J. Phys.Cond. Matter. 16, S4439 (2004).

[6] T. Schneider et al., Phys. Rev. Lett. 86, 4899 (2001).

[7] A. Bussmann-Holder, et al., Europhys. Lett., 72, 423, (2005).

[8] V. Loktev et al., J. Low T Phys. 143, 115 (2006); Xiao-Jia Chenet al., J. Phys.: Cond. Matter 19, 425236 (2007).

[9] G. M. Zhao, et al. Phys. Rev. B 63, 220506(R) (2001).

[10] M. H. Hettler et al., Phys. Rev. B 58, R7475 (1998); 61, 12739 (2000).

[11] Th. Maier et al., Rev. Mod. Phys. 77, 1027 (2005).

[12] M. Jarrell et al., EuroPhy. Lett., 56, 563 (2001).

[13] P. W. Anderson, The Theory of Superconductivity in the 
High- $T_{c}$ Cuprates, Princeton University Press, Princeton, NJ (1997).

[14] F. C. Zhanget al., Phys. Rev. B 37, 3759 (1988).

[15] A. Macridin et al., Phys. Rev. Lett. 97, 056402, (2006).

[16] A. Macridin et al., cond-mat/0611067.

[17] G. V. M. Williams et al., Phys. Rev. Lett. 80, 377 (1998).

[18] G. M. Zhao et al., Phys. Rev. B 50, 4112 (1994).

[19] A. Lanzara et al., Nature (London) 412, 510 (2001); T. Cuk et al., Phys. Status Solidi (b) 242, 11 (2005).

[20] M. Jarrell et al., Phys. Rev. B 64, 195130 (2001).

[21] M. Jarrell et al., Physics Reports 269 No.3, 133 (1996).

[22] $\lambda_{c}$ and the CRO region are dependent on the phonon mode, e.g. in [16] we find $\lambda_{c} \approx 0.25$ and a narrower CRO region for the buckling mode.

[23] Th. Maier et al., Phys. Rev. B 74, 094513 (2006).

[24] Ad Lagendijk et al., Phys. Lett. A 108, 91 (1985); V. V. Kabanov et al., Phys. Rev. B 47, 6060 (1993).

[25] A. Shengelaya et al. Phys. Rev. Lett. 83, 5142 (1999).

[26] R. Khasanov et al. Phys. Rev. Lett. 92, 057602 (2004); J. Hofer et al. Phys. Rev. Lett 84, 4192 (2000); G. M. Zhao et al., Phys. Rev. B 51, 16487 (1995).

[27] A. Lanzara, et al. J. Phys. Cond. Matter 11, L541 (1999).

[28] V. J. Emery, S. A. Kivelson, Nature 374, 434 (2002). 